Loew: Klarst. d. Bez. zwisch. Formose u. Methylenitan. 203

\title{
Zur Klarstellung der Beziehungen zwischen Formose und Methylenitan;
}

\author{
von \\ O. Loew.
}

$\mathrm{Da}$, wie aus dem neuesten Hefte von Liebig's Annalen ${ }^{1}$ ) hervorgeht, Tollens der Meinung ist, dass Formose und Methylenitan identisch seien, so sehe ich mich nochmals gezwungen, diesen mir unbegreiflichen Irrthum in ein klares Licht zu stellen, und werde dabei möglichst kurz und bündig verfahren.

I. Tollens erhielt durch Umwandlung von Formaldehyd durch Aetzbaryt ${ }^{2}$ ) bei $55^{\circ}-60^{\circ}$ einen gelben, zum Theil in Aether löslichen Körper, welcher, drei Stunden bei $100^{\circ}$ getrocknet, der Formel $\mathrm{C}_{6} \mathrm{H}_{10} \mathrm{O}_{5}$ entsprach. Ich habe diese Versuche wiederholt, kann diese Angaben bestätigen und noch hinzufügen, dass dieses Produkt braun ist und sehr bitter schmeckt.

II. In neuester Zeit hat Tollens nach meinen Angaben mit Kalk bei gewöhnlicher Temperatur die Condensation des Formaldehyds vorgenommen, aber das Produkt 30 Stunden bei $90^{\circ}$ getrocknet und findet statt im Mittel $44,4 \% \mathrm{C}$ wie oben, nun im Mittel $42,6 \% \mathrm{C}$ und will daraus die Formel $\mathrm{C}_{12} \mathrm{H}_{22} \mathrm{O}_{11}$ ableiten. - Hierzu bemerke ich, dass in Folge der sehr labilen Beschaffenheit der Formose bei diesem lange fortgesetzten Trocknen bei $90^{\circ}$ der ursprünglich süsse Geschmack in einen bittern umschlägt, woraus hervorgeht, dass der hierbei stattfindende Gewichtsverlust nicht das hygroskopische Wasser allein betrifft, sondern dass eine Zersetzung unter Wasserverlust eintritt. Hätte Tollens sich die geringe Mühe genommen und die Reductionsfähigkeit seines getrockneten Produktes für Fehling's Lösung bestimmt, so würde er gefunden haben, dass diese schon um über die Hälfte vermindert war. Nachdem ich bei meinen Arbeiten über Formose vor zwei Jahren ihre

1) Ann. Chem, 243, 334.

2) Ber. 15, 1633 u. 16, 919 . 


\section{Loew: Zur Klarstellung der Beziehungen}

leichte Zersetzlichkeit beobachtet hatte, trocknete ich möglichst lange im Vacuum über Schwefelsäure und dann schliesslich noch kurze Zeit bei $80^{\circ}-85^{\circ}$. Will man längere Zeit bei höherer Temperatur trocknen, so sollte man $70^{\circ}$ nicht überschreiten. Tollens analysirte somit wieder einen zersetzten Zucker, diesmal war die Hauptzersetzung erst beim Erhitzen herbeigeführt worden, das erste Mal aber schon durch das heisse Barytwasser bei der Bereitung. Das unzersetzte Produkt gab mir im Mittel 40,2\% C, entsprechend der Formel $\mathrm{C}_{6} \mathrm{H}_{12} \mathrm{O}_{6}{ }^{1}$ ). Würde die Formose ein recht beständiger Körper sein und in möglichst grossen Krystallen anschiessen, so wäre sie allerdings längst entdeckt worden und wohl nie ein solcher Streit darüber entstanden.

III. Tollens meint, da der kaustische Kalk schon in der Kälte auf Formose bald einwirke, wäre es ganz unmöglich, die Gelbfärbung, d. h. die Wiederzersetzung der Formose kurz nach ihrer Bildung zu verhindern. Hierauf ist zu erwidern, dass bei der nöthigen Sorgfalt der richtige Zeitpunkt wohl beobachtet werden kann. Ich habe stets die Vorsicht gebraucht, die Condensationsmischung auf 5-6 Flaschen zu vertheilen, damit nicht Alles verloren war, im Falle in einer Flasche bereits Gelbfärbung d. h. beginnende Wiederzersetzung eingetreten war. ${ }^{2}$ ) Nur die ganz farblos gebliebenen Lösungen dürfen auf Formose verarbeitet werden. Ich habe ferner früher erwähnt ${ }^{3}$ ), dass man bei der nöthigen Vorsicht auch das Gelbwerden beim Einengen der Formoselösung wohl verhindern kann; besonders

1) Es sei hier anch auf den Umstand hingewiesen, dass Wehmer, der Mitarbeiter von Tollens, mittheilt, dass auch seine Analysen auf diese Formel stimmen. (Bot. Ztg. 1887. Nr. 44.)

$\left.{ }^{2}\right)$ Ich labe derlei praktische Handgriffe in meiner ursprünglichen Abhandlung (Dies. Joum. [2] 33, 321) nicht angeführt, weil ich vermuthete, dass Chemiker, die vertraut mit dem Umgehen mit labilen Stoffen sind, von selbst leicht darauf kommen würden. Da jene Gelbfärbung beim Contact mit Basen als Zeichen der Condensation des Formaldehyds von manchen angesehen wurde, so begreift sich, dass man stets nur zersetzte Formose erhalten hatte.

3) Ber. 20, 142 . 
leicht ist dieses, wenn die Oxalsäure bis zur deutlich sauren Reaction zugesetzt wird.

IV. Tollens hat das Osazon der Formose nicht darstellen können, was mir ein klarer Beweis ist, dass er wieder mit zersetzter Formose operirte; denn dasselbe scheidet sich - allerdings weit langsamer als bei Dextrose - nach 3-4 stündiger Digestion auf dem Wasserbade zuerst ölig aus, und giebt beim Reinigen mit Benzol und Umkrystallisiren aus Alkohol schön gelbe Nadeln. ${ }^{1}$ ) Auch hier ist Tollens mit einer späteren Mittheilung ${ }^{2}$ ) seines Mitarbeiters Wehmer in offenbarem Widerspruche; denn letzterer hat das Osazon erhalten und auch den Schmelzpunkt richtig bestimmt. ${ }^{3}$ )

V. Das von Tollens erhaltene „,Methylenitan" hat eine Reductionskraft von $1 / 6-1 / 4$ derjenigen der Dextrose, während das der Formose nahezu dem der Dextrose gleich ist. Da fractionirte Fällungen der Formose mit Alkohol Produkte mit nur äusserst geringem Unterschied im Reductionsvermögen und Osazone von gleichem Schmelzpunkte lieferten, so glaube ich im Rechte zu sein, die Behauptung von Tollens, es lägen Gemenge vor, zurückzuweisen.

VI. Da nun Formose süss schmeckt, das Methylenitan aber bitter, da Formose Fehling's Lösung viermal stärker reducirt als das „Methylenitan“, da Formose ein Osazon liefert, das Methylenitan aber nicht, da ferner die Zusammensetzung der Formose der Formel $\mathrm{C}_{6} \mathrm{H}_{12} \mathrm{O}_{6}$ entspricht, die des Methylenitans aber der Formel $\mathrm{C}_{6} \mathrm{H}_{10} \mathrm{O}_{5}$, da ferner Formose beim Kochen mit kohlensaurem Kalk Kohlensäure nicht austreibt, wohl aber das Methylenitan, letzteres also eine Lactonnatur ${ }^{4}$ ) verräth, so wird jeder billig Denkende mit mir einverstanden sein, wenn ich

1) Rascher erhält man es beim Eindampfen der Reactionsmischung bis zum Syrup.

2) Der Tollens'sche Artikel ist offenbar früher verfasst, als der Wehmer'sche.

$\left.{ }^{3}\right)$ Ber. 20, 1617.

4) Ich habe früher bereits darauf hingewiesen, dass das „Methylenitan" als Hauptprodukt der Formosezersetzung einen saccharinartigen Charakter trägt. 
206 Loew: Klarst. d. Bez. zwisch. Formose u. Methylenitan.

energisch gegen den Vorschlag ron Tollens protestire, die Formose ebenfalls mit dem Namen Methylenitan zu belegen. Es ist bis jetzt nicht Usus gewesen, eine Substanz mit demselben Namen zu belegen, wie ihre Zersetzungsprodukte.

VII. Tollens behauptet, ich hätte einen Rückzug angetreten, wenn ich erklärte, die Formose, obgleich ein wahrer Zucker, habe keine engeren Beziehungen zur Dextrose. Dieser Meinung war ich von Anfang an und habe dieselbe auch vom Sorbin und Eucalyn. Einer anderen Meinung hätte ich erst werden können, wenn eine Ueberführung in Dextrose oder Mannit geglückt wäre. Uebrigens verweise ich hier auf meine Auseinandersetzungen in den Berichten. ${ }^{1}$ )

VIII. Tollens weist die Behauptung zurück, dass Galactose mit Bierhefe nicht gährungsfähig sei. Indessen diese rührt nicht von mir selbst her, denn ich habe mich nie mit Galactose beschäftigt, sondern von Koch, der mit Nachdruck darauf hinwies ${ }^{2}$ ), so dass ich es für richtig hielt.

IX. Tolle ns nennt das Verfahren, mit erhitztem Kupferdraht Formaldehyd darzustellen, se in Verfahren. Mit welchem Rechte wird aus dem Hinweis klar, dass er früher mit Platin nur 2-3 procentigen Aldehyd in einer Operation herstellen konnte, ich aber das Kupfer in Vorschlag brachte, das mir einen solchen von $16 \%-20 \%$ lieferte. Seine Verbesserungen bestanden darin, dass er den Apparat viel mehr complicirte, den Methylalkohol gelinde erwärmte und Glimmerplättchen zum Einhüllen der Kupferspirale vorschlug, um das Springen der Röhre zu verhindern. Letzteren Zweck erreiche ich einfacher dadurch, dass ich gutes bölmisches Glas nehme. Indessen hierüber einen Prioritätsstreit zu entflammen, liegt mir fern; möge Tollens fortfahren, die Methode mit Kupfer als die seine zu bezeichnen.

München, Pflanzenphysiol. Inst., Februar 1888.

1) Ber. 20, 3040 .

2) Dass. 20, Ref. 145. Beilstein (1881) und Richter (1882) führen in ihren Werken ebenfalls an, dass Galactose nicht gährungsfähig sei. 